\title{
Analysis of HIPPARCOS Measurements: Positions, Proper motions, Parallaxes
}

\author{
H.G. Walter ${ }^{1}$, M. Froeschlé2 , J.L. Falin ${ }^{2}$, R. Hering ${ }^{1}$, \\ J. Kovalevsky ${ }^{2}$, H. Lenhardt ${ }^{1}$
}

(1) Astronomisches Rechen-Institut

Mönchhofstrasse 12-14

D-6900 Heidelberg, Germany

(2) Observatoire de la Côte d'Azur

CERGA

Avenue Copernic

F-06130 Grasse, France

\begin{abstract}
Using the first 12 months of HIPPARCOS mission data already reduced, a synthesis solution was obtained by both CERGA and ARI teams. The reduction includes two steps. In the first one the origins of individual reference circles are determined, thus constituting the HIPPARCOS celestial reference frame. This solution uses a subset of some 30000 bright and evidently single stars in order to minimize possible corruptive effects on the system. In the second step, the abscissa measurements of all observed stars are substituted in the system and then processed star by star in a least squares adjustment providing the astrometric parameters (positions and parallaxes).

The results of sphere solution and astrometric parameter determination are discussed. In this context error ellipses and internal errors are calculated. External errors are derived by comparison with suitable astrometric catalogues. Especially, for estimating the external errors of the HIPPARCOS measured FK5 stars the instrumental system was tied to the FK5 system by rigid rotations followed by comparisons of the positions with those of FK5. The mean internal errors of the HIPPARCOS positions of FK5 stars is 1.5 milliarcseconds (mas) while the standard deviation of the differences HIPPARCOS minus FK5 reaches 90 mas. For the 5022 measured parallaxes the mean internal and external errors are 3.8 mas and 16.7 mas, respectively. - The results of precision and comparisons with external data show that the accuracy predicted for the final catalogue can be reached.
\end{abstract}

\section{Introduction}

Launched in August 1989 the astrometry satellite HIPPARCOS has entered the operational phase in December 1989. Since then it is collecting regularly data of which about $70 \%$ of useful data are recovered owing to limitations of the telemetry network. We deal here with the analysis of 12 months of data performed within the data reduction consortium FAST. The data are acquired between Dec. 1989 and Dec. 1990 and form the basis for the determination of astrometric parameters, i.e. the two components each of the positions and the trigonometric parallaxes of the HIPPARCOS programme stars. Two steps are emphasized: (1), the sphere solution which establishes the HIPPARCOS celestial reference frame and (2), the determination 
and validation of astrometric parameters star by star in the routine and selective mode.

A considerable amount of preprocessing of raw measurements is required before sphere solution and astrometric parameter determination can ultimately be supplied with the essential "observables" which are the star abscissae on reference great circles (RGC's). Details on the data streams, their interlacing and the procedures used are laid down in Kovalevsky et al. (1992).

In the following sections we present the results of sphere solution and astrometric parameter determination. They are based on 1226986 star abscissae on 758 RGC's. Statistical tests are applied to assess the internal errors of the parameter solution in a global and individual manner. In addition, external errors are derived from comparison with available accurate ground-based astrometric quantities. For taking account of possible discrepancies of the reference frames either the angles describing a rigid rotation between coordinate systems are calculated, or differential techniques are used free of any hypotheses of the frame definitions.

\section{Sphere solution}

The abscissae $\eta$ obtained on a given reference great circle are not absolute as there remains an indeterminate rotation about its axis. The origin of abscissae is the one that minimizes the norm of the vector of residuals. Thus, the first task before attempting the determination of astrometric parameters is to compute the corrections to the origins of individual circles so that they form a coherent set of coordinate systems in which the projections of a star appearing on several great circles are consistent.

Let us consider one star whose approximate astrometric parameters, expressed in the ecliptic coordinate system, are $\lambda_{0}, \beta_{0}, \mu_{\lambda 0} \cos \beta_{0}, \mu_{\beta 0}, \pi_{0}$ and let $\Delta \lambda, \Delta \beta, \Delta \mu_{\lambda} \cos \beta, \Delta \mu_{\beta}, \Delta \pi$ be the unknown corrections to be applied in order to get the actual parameters. Knowing the orientation of the RGC, one can express the abscissa $\eta=\eta_{0}+\Delta \eta$ of the star as a linear function of the unknown corrections where $\eta_{0}$ is the abscissa inferred from the approximate parameters. In reality, one has to add the correction $\Delta \omega$ of the origin to the measured abscissa. We also add some further unknowns representing a possible distortion of the abscissa scale that might be due to external systematic effects, such as a colour error and thermal variation of the basic angle depending on the difference between the abscissa $\eta$ of the star and $\eta_{s}$ of the Sun. Let $\Gamma_{k}(\mathrm{k}=1$ to 8 ) be these additional parameters. If index $\mathrm{i}$ refers to a star and $\mathrm{j}$ to the $\mathrm{RGC}$, the equation of condition has the following form

$$
\Delta \eta_{i j}=\mathrm{a}_{i j} \Delta \lambda_{i}+\mathrm{b}_{i j} \Delta \beta_{i}+\mathrm{a}_{i j}^{\prime} \Delta\left(\mu_{\lambda} \cos \beta\right)_{i}+\mathrm{b}_{i j}^{\prime} \Delta \mu_{\beta_{i}}+\mathrm{c}_{i j} \Delta \pi_{i}+\Delta \omega_{j}+\sum_{k=1}^{8} \frac{\partial \eta_{i j}}{\partial \Gamma_{k}} \Delta \Gamma_{k} .
$$

One writes as many such equations as there are star abscissae available. In order to get a reliable determination of $\Delta \omega_{j}$ a sufficient number of equations for each star is necessary so that the corrections to the astrometric parameters can be safely eliminated. - Initially we set $a_{i j}^{\prime}=$ $\mathrm{b}_{i j}^{\prime}=\mathrm{c}_{i j}^{\prime}=0$. This provides sufficient equations to determine $\Delta \omega_{j}$ but introduces a bias due to unknown corrections to proper motions and parallaxes.

The determination of origins yields standard deviations well below 0.5 milliarcseconds (mas), the dispersion being of the order of 20 mas. This gives the order of magnitude of the precision with which the reference system is built. 
The eight general calibration parameters $\Gamma_{k}$ are the following:

$$
\begin{aligned}
\cos \left(\eta-\eta_{s}\right) & =-0.0082 \mathrm{mas} \\
\cos 2\left(\eta-\eta_{s}\right) & =+0.0146 \\
\sin 2\left(\eta-\eta_{s}\right) & =-0.0838 \\
\cos 3\left(\eta-\eta_{s}\right) & =+0.2833 \\
\sin 3\left(\eta-\eta_{s}\right) & =+0.4378 \\
\cos 6\left(\eta-\eta_{s}\right) & =+0.4877 \\
\sin 6\left(\eta-\eta_{s}\right) & =-0.4260 \\
\mathrm{~B}-\mathrm{V}-0.5 & =-2.1491 \mathrm{mas} / \mathrm{mag}
\end{aligned}
$$

The $\sin \left(\eta-\eta_{s}\right)$ term is not introduced. Because of its proportionality to the effect of the parallax on the star abscissa it is inseparable from $\Delta \pi$.

\section{Astrometric parameter determination}

The application of the origin correction to the abscissae completes the input data necessary for the determination of astrometric parameters. Methods and algorithms for this task are laid down in Walter et al. (1985); the computations are performed with reference to the ecliptic coordinate system at $\mathrm{J} 2000.0$.

The short span of 12 months of data suggests to confine the parameter determination in the first instant to the two components of the position, i.e. ecliptic longitude, latitude, and the trigonometric parallax. During the 12 months interval 118323 stars have been observed, the observations of which are distributed over 758 RGC's. We restrict ourselves here to the global results of the three parameter solution listed in Table 1 .

Table 1. Statistics and error budget of the three parameter solution at Jul. epoch 1992.0

Number of stars having successful solution: $\mathrm{N}=112444$

Average number of useful observations per star: $\quad 9.1$

Average number of outliers: $\quad 1.5$

Average a posteriori (a priori) error of unit weight: 14.5 mas (10.0 mas)

Average semi-major axis of error ellipses: $\quad 3.4$ mas

Average semi-minor axis of error ellipses: $\quad 1.8 \mathrm{mas}$

Average standard deviations:

$$
\begin{aligned}
& \bar{\sigma}_{\lambda}=\frac{\Sigma \sigma_{\lambda} \cos \beta}{N}=3.0 \pm 0.1 \mathrm{mas} \\
& \bar{\sigma}_{\beta}=\frac{\Sigma \sigma_{\beta}}{N}=2.4 \pm 0.1 \mathrm{mas} \\
& \bar{\sigma}_{\pi}=\frac{\Sigma \sigma_{\pi}}{N}=3.8 \pm 0.1 \mathrm{mas}
\end{aligned}
$$

Owing to the measurement technique correlations between positions and parallaxes are expected. Their magnitude, however, is surprisingly low with a view to the limited data yield of 12 months: For $75 \%$ of the parameter solutions the correlation coefficients lie in the small band of -0.4 to +0.4 . 


\section{Qualification tests}

'To assess the quality of the results we followed two principal lines: (1), comparison with a reference catalogue (RC) in ecliptic coordinates derived from the Input Catalogue (Turon et al., 1992) in order to identify possible large scale departures from the reference catalogue, and (2), the results are compared with ground-based astrometric data of the best possible quality, ultimately aiming at the estimation of external errors.

\subsection{Comparison with the Reference Catalogue}

The comparison of the astrometric parameters with the Reference Catalogue is given in Table 2. Here, a star is considered an outlier and omitted when $|\Delta \lambda \cos \beta|,|\Delta \beta|$ is greater than 1 arcsec or $|\Delta \pi|$ is greater than 100 mas.

Table 2. Results of 12 months data compared with the Reference Catalogue at Jul. epoch 1992.0, HIP minus RC

\begin{tabular}{lccc} 
& $\begin{array}{c}\Delta \lambda \cos \beta \\
{[\mathrm{mas}]}\end{array}$ & $\begin{array}{c}\Delta \beta \\
{[\mathrm{mas}]}\end{array}$ & $\begin{array}{c}\Delta \pi \\
{[\mathrm{mas}]}\end{array}$ \\
\hline Number of stars & \multicolumn{2}{c}{108533} & 5022 \\
$\begin{array}{l}\text { Unweighted } \\
\text { mean difference }\end{array}$ & $1.6 \pm 0.9$ & $51.8 \pm 0.9$ & $0.3 \pm 0.2$ \\
RMS difference & 307.5 & 308.3 & 16.7 \\
Standard deviation & 307.5 & 303.9 & 16.7 \\
\hline
\end{tabular}

The RMS differences in Table 2 reflect essentially the position accuracies of the Reference Catalogue for which about 300 mas are quoted. Also, the errors of the parallax differences seem to be free from significant portions caused by the HIPPARCOS measurements since the value of about 17 mas is nearly equal to the mean error ( 15 mas) of the Catalogue of Trigonometric Parallaxes (Jenkins, 1952, 1963), from which most of the parallaxes of the Reference Catalogue are drawn.

\subsection{Comparison with FK5}

At present, 1418 stars of the FK5 (Fricke et al., 1988) could be identified among the available data, two of them are to be discarded because of spurious errors. Calculating for each star the position differences HIP-FK5, we allow for systematic differences between our 12 months solution and the FK5 frame by simply fitting the three angles of a rigid rotation about the $\mathrm{x}$-, $y$ - and $z$-axis of the ecliptic coordinate system to the position vectors referring to the respective frames. Some statistics of the comparison are displayed in Table 3.

From the errors quoted in FK5 one would expect standard deviations of the differences of 50 to 60 mas taking into account systematic and individual errors. Part of the surplus may be explained by imprecisions of the FK5 proper motions. Fig. 1 shows the regional distribution of the difference vectors HIP-FK5 indicating stronger systematic deviations in the southern than in the northern hemisphere. 
Independence of the coordinate frames is achievable by resorting to abscissa differences of pairs of FK5 stars along an RGC. Bringing into play all combinations of two stars on a selected RGC the HIPPARCOS measured abscissa differences $(\Delta \mathrm{HIP})$ and the abscissa differences calculated

Table 3. Statistics and error budget of the HIPPARCOS results compared with FK5 positions at epoch 1992.0, HIP minus FK5

Number of stars in the sample: $\mathrm{N}=1416$

Average standard deviations:

$\bar{\sigma}_{\lambda}=1.6 \mathrm{mas}, \bar{\sigma}_{\beta}=1.4 \mathrm{mas}$

Angles of rotation into the FK5 frame:

$\begin{aligned} & \phi_{x}=-7.8 \pm 3.0 \mathrm{mas} \\ & \phi_{y}=2.2 \pm 3.0 \mathrm{mas} \\ & \phi_{z}=61.7 \pm 3.2 \mathrm{mas}\end{aligned}$

\begin{tabular}{lcc} 
After rotation: & $\begin{array}{c}\Delta \lambda \cos \beta \\
{[\mathrm{mas}]}\end{array}$ & $\begin{array}{c}\Delta \beta \\
{[\mathrm{mas}]}\end{array}$ \\
\hline $\begin{array}{l}\text { Unweighted } \\
\text { mean difference }\end{array}$ & $+1.7 \pm 2.4$ & $+38.5 \pm 2.4$ \\
Standard deviation & 90.3 & 90.3 \\
\hline
\end{tabular}

from the FK5 catalogue ( $\triangle \mathrm{FK} 5)$ are set up for the respective epoch of observation to yield the quantity $\Delta=\Delta \mathrm{HIP}-\Delta \mathrm{FK} 5$ for each pair as a function of the abscissa difference.

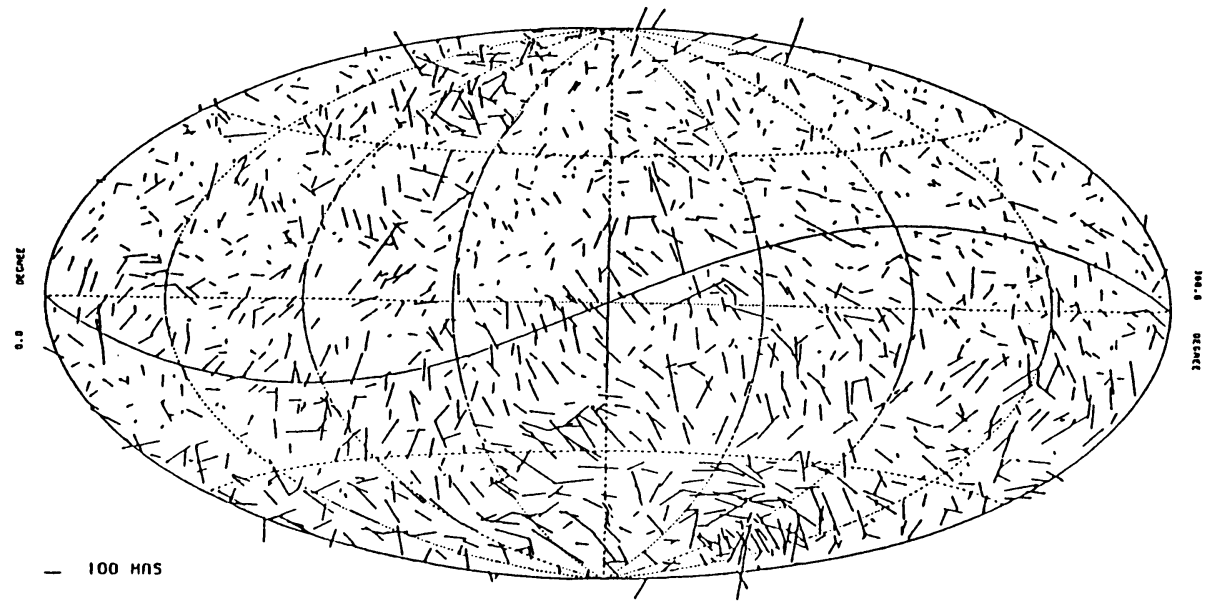

Fig. 1. Difference vectors, HIP-FK5, in the ecliptic coordinate system; tracing of the celestial equator. The arrows point from the FK5 position to the HIP position after rotation.

On determining mean differences, and standard deviations of the $\Delta$ 's per RGC one gets a 
measure of accuracy in terms of relative abscissa measurements. As an example, we present in Fig. 2 a plot of the $\Delta$ 's belonging to RGC No. 237 whose pole is defined by $\lambda_{P}=3.6^{\circ}$ and $\beta_{P}=$ $-41.9^{\circ}$. From the $19 \mathrm{FK} 5$ stars, i.e. 171 pairs along this RGC, one finds for the mean difference 30.4 mas and for the standard deviation 119.3 mas.

Other spot checks resulted in distribution patterns of $\Delta$ similar to Fig. 2 and errors of the size above while the nominal FK5 accuracy and an assumed precision of a few mas of the abscissae would predict standard deviations below 90 mas. Again, the FK5 proper motions may be responsible for the excess.

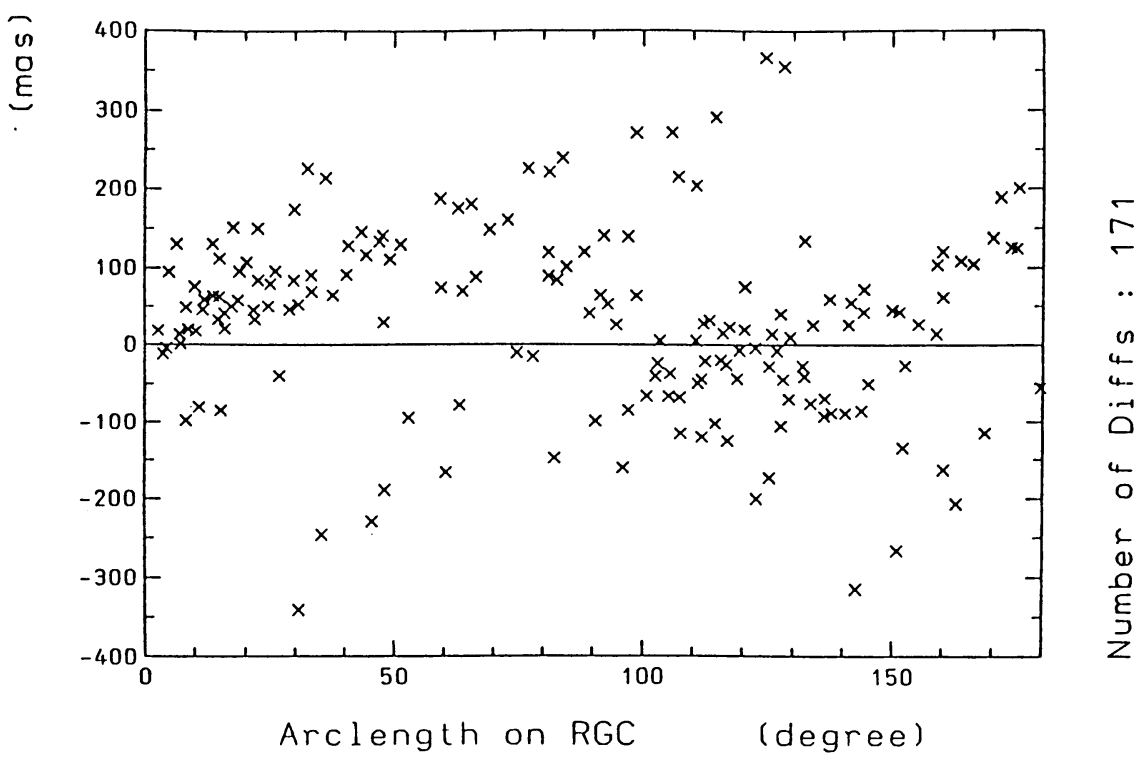

Fig. 2. Differences (HIP-FK5) of the HIPPARCOS measured arcs and the arcs calculated from FK5 versus arclength for all pairs of FK5 stars on RGC No. 237 at Jul. epoch 1990.196

\subsection{Comparison with ground-based trigonometric parallaxes}

We have compared the HIPPARCOS parallaxes with the Catalogue of Nearby Stars, CNS3 (Gliese and Jahreiss, 1991). If the comparison is confined to stars within $25 \mathrm{pc}$ distance from the Sun, we find 1231 stars in common. Their parallaxes are plotted in Fig. 3 pointing to a possible selection effect among those nearby stars in CNS3 which lie close to the distance limit of 25 pc. Different distributions of the CNS3 parallaxes and the HIPPARCOS measured parallaxes of CNS3 are found. Above all a strong decrease of the parallaxes around 40 mas is noticeable.

\section{Conclusion}

By comparing the astrometric parameters deduced from 12 months of HIPPARCOS observations with positions and parallaxes of the Reference Catalogue it was possible to reproduce its nominal 
mean errors. The RMS differences, therefore, give evidence that the HIPPARCOS positions and parallaxes assume reliable values and that the corrections of the parameter adjustment are of the order of the accuracies of the Reference Catalogue. - Some checks using ground-based astrometric data of high quality - such as FK5 stars and parallaxes of nearby stars - demonstrate external accuracies below the 10 mas level with the data now available thus indicating that the specified precision of the final catalogue will be reached when more data have arrived.

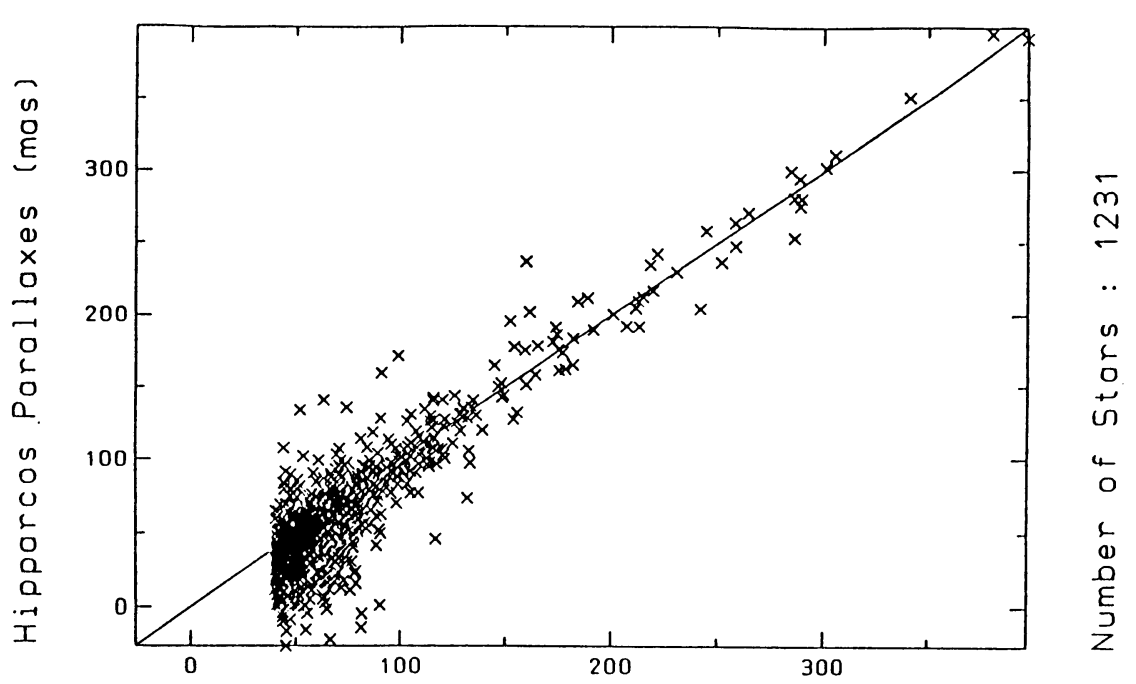

Fig. 3. HIPPARCOS parallaxes versus the parallaxes of the Catalogue of Nearby Stars.

\section{Acknowledgements}

Some of the authors (H.G.W., R.H., H.L.) are indebted to the financial support by the Bundesministerium für Forschung und Technologie (BMFT), Bonn, Germany, under the Project No. 500090020 .

\section{References}

Fricke, W. et al., 1988, Fifth Fundamental Catalogue (FK5), Verlag G. Braun, Karlsruhe.

Gliese, W., Jahreiss, H., 1991, Catalogue of Nearby Stars, 3rd Edition, in: The Astronomical Data Center CD-ROM, Selected Astronomical Catalogs, Vol. I, Doc. No. STX-T-1-5002009-91, L.E. Brotzman and S.E. Gessner (eds.), NASA, Goddard Space Flight Center, Greenbelt, MD 20771.

Jenkins, L.F., 1952, General Catalogue of Trigonometric Stellar Parallaxes, Yale University Observatory, New Haven, Conn.

Jenkins, L.F., 1963, Supplement to the Catalogue of Trigonometric Stellar Parallaxes, Yale Univ. Obs., New Haven, Conn. 
Kovalevsky, J. et al., 1992, Astron. Astrophys. 258, 7.

Turon, C. et al., 1992, The Hipparcos Input Catalogue, ESA Publication Division, c/o ESTEC, Noordwijk, ESA SP. 1136.

Walter, H.G., Hering, R., Bastian, U., Bernstein, H.-H., 1985, Astrometric parameter determination: Methods, algorithms and program implementation. Proc. Marseille Coll. 'Processing of Scientific Data from Hipparcos', Second FAST Thinkshop, 21 - 25 Jan. 1985, J. Kovalevsky (ed), 281. 